\title{
Numerical Modelling on the Influence of Source in the Heat Transformation: An Application in the Metal Heating for Blacksmithing
}

\section{H. P. Kandel, J. Kafle and L. P. Bagale}

Journal of Nepal Physical Society

Volume 7, Issue 2, June 2021

ISSN: 2392-473X (Print), 2738-9537 (Online)

Editors:

Dr. Binod Adhikari

Dr. Bhawani Joshi

Dr. Manoj Kumar Yadav

Dr. Krishna Rai

Dr. Rajendra Prasad Adhikari

Mr. Kiran Pudasainee

JNPS, 7 (2), 97-101 (2021)

DOI: https://doi.org/10.3126/jnphyssoc.v7i2.38629

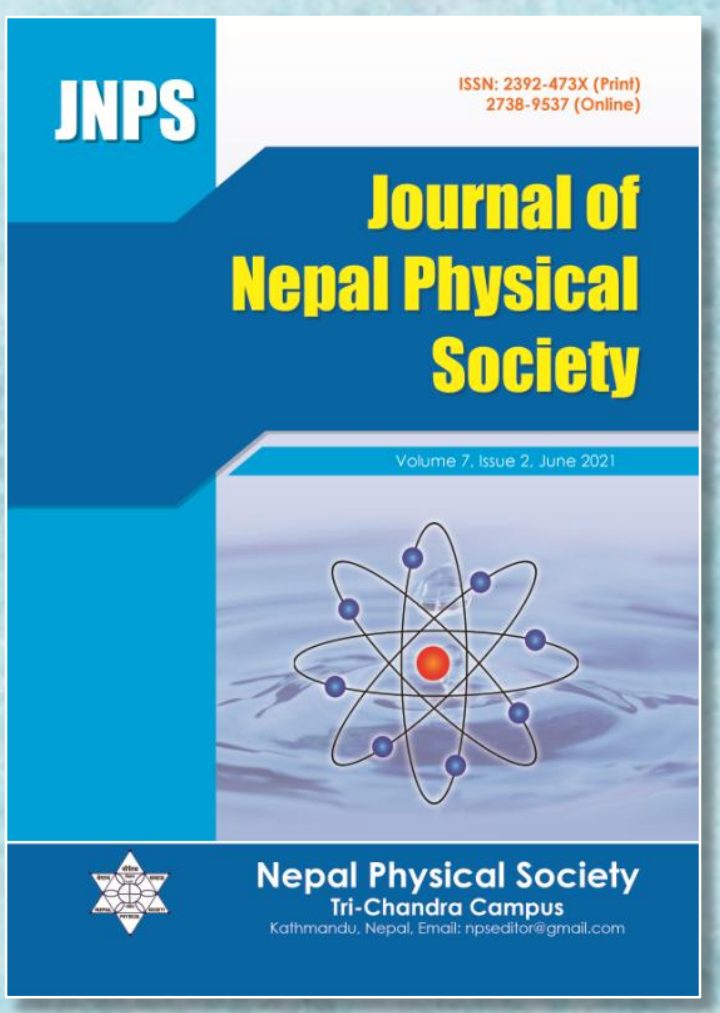

Published by:

Nepal Physical Society

P.O. Box: 2934

Tri-Chandra Campus

Kathmandu, Nepal

Email: nps.editor@gmail.com 


\title{
Numerical Modelling on the Influence of Source in the Heat Transformation: An Application in the Metal Heating for Blacksmithing
}

\author{
H. P. Kandel ${ }^{1,2}$, J. Kafle ${ }^{2}$ and L. P. Bagale ${ }^{2}$ \\ ${ }^{1}$ Golden Gate International College, Buttisputali, Kathmandu, Nepal \\ ${ }^{2}$ Central Department of Mathematics, Tribhuvan University, Kirtipur, Kathmandu, Nepal \\ Corresponding Email: jeevan.kafle@cdmath.tu.edu.np
}

Received: 16 March, 2021; Revised: 13 April, 2021; Accepted: 15 June, 2021

\begin{abstract}
Many physical problems, such as heat transfer and wave transfer, are modeled in the real world using partial differential equations (PDEs). When the domain of such modeled problems is irregular in shape, computing analytic solution becomes difficult, if not impossible. In such a case, numerical methods can be used to compute the solution of such PDEs. The Finite difference method (FDM) is one of the numerical methods used to compute the solutions of PDEs by discretizing the domain into a finite number of regions. We used FDMs to compute the numerical solutions of the one dimensional heat equation with different position initial conditions and multiple initial conditions. Blacksmiths fashioned different metals into the desired shape by heating the objects with different temperatures and at different position. The numerical technique applied here can be used to solve heat equations observed in the field of science and engineering.
\end{abstract}

Keywords: Partial differential equation, Heat equations, Parabolic equations, Finite difference methods, Numerical solutions, Metal Heating, Blacksmithing.

\section{INTRODUCTION}

A partial differential equation (PDE) involves partial differential coefficients, i.e., the partial derivative of a dependent variable with more than one independent variable. As there is always more than one independent variable in a physical problem. PDEs are important in many branches of science and engineering. We can solve only a few PDEs, mostly linear equations and some nonlinear equations $[1,2,8,10]$. The heat equation, also known as the diffusion equation in mathematics and physics, is a partial differential equation (PDE) that describes the distribution of heat evolution over time in a solid medium $[2,8,10]$. The heat equation is an important PDE which describes the variation in temperature (or distribution of heat) in a given region over time (Fig. 1). The heat equation (Diffusion equation) is widely used in particle diffusion, Brownian motion, Schrodinger equation for a free particle and thermal diffusivity in polymers. It's also used in metal processing industry, the outer surface of rockets, railway tracks and bridges, refrigerators, image analysis, cancer modeling and spatial ecological modeling. As a result, the heat equation is extremely important in a variety of scientific fields $[2,4,5,8,9,10,24]$.

Let $u=u(x, t)$, then the partial differential equation of the form

$$
u_{t}(x, t)=c u_{x x}(x, t), c>0 \ldots(1.1)
$$

where $c$ is called thermal diffusivity, is one dimensional heat equation (diffusion equation). It is an example of a prototypical parabolic partial differential equation $[1,4]$.

In the regular shape domain, the heat equation has an analytic solution, whereas in the irregular shape domain, computing analytic solution of such equations is very difficult $[12,19]$. As a result, we use numerical methods to compute the solution of the modeled partial differential equations. We use the finite difference method (FDM) to find numerical solutions to heat transfer problems, which was 
introduced by Brook Taylor in 1715 and has been studied as abstract self-standing mathematical objects in works by George Boole (1860), C.M. Milne Thomson (1933), and Karaly Jordan (1939) [12, 19].

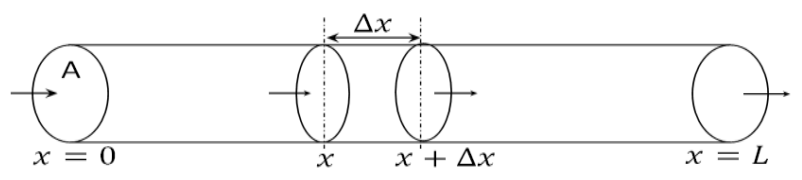

Fig. 1: Heat flow in a rod. Source....

By discretizing the domain into a finite number of regions, we can compute the numerical solution of partial differential equations. The solutions are computed at the domain's grid points $[12,19]$. The numerical solutions of a one dimensional heat equation together with an initial condition and boundary conditions using finite difference methods does not always converge to the exact solutions $[12,19]$. It denotes numerical instability in finite difference methods.

A blacksmith is a metalworker who fashions various objects out of iron or steel. By heating, hitting, twisting, and cutting metals, blacksmiths create objects such as gates, grilles, railings, light fixtures, furniture, sculpture, tools, agricultural implements, decorative and religious items, cooking utensils, and weapons. During their work, blacksmiths heat the metals to soften them so that they can be shaped with hand tools such as hammers, chisels, anvils, and so on. They estimate the temperature distribution on their metal pieces by looking at the glowing color on them. Heat may be applied to single or multiple locations on the metal [22].

We are all aware that heat transfers from a hotter body to a colder body. Temperature change is a major effect of heat transfer: heating raises the temperature while cooling lowers it. We assume that there is no phase change and that no work is done on or by the system throughout our work. Experiments show that the transferred heat is affected by three factors: temperature change, system mass, and substance phase [7, 17, 23]. In practice, we must apply different amounts of heat to different parts of the material in order to change the metals into the desired shape. One of the prominent examples is a blacksmith working on hot iron which is shown in Fig. 2.

Chamkha and Khaled [3] investigated the effect of magnetic field on coupled heat and mass transfer by mixed convection in a linearly stratified stagnation flow in the presence of internal heat generation or absorption. Exact analytic solutions and modeling for hydro magnetic oscillatory rotating flows of an incompressible Burgers fluid bounded by a plate [5, 16, 18]. Jean Baptise Joseph Fourier (1768-1830) invented the heat equation, which he presented as a manuscript to the Institute de France in 1807 AD and published in his monograph, Analytic Theory of Heat, in $1822 \mathrm{AD}$ [13]. Scientists in the 1600s appeared to have been correct in their belief that heat is related to the motion of microscopic constituents of matter. However, in the 1700s, it was thought that heat was a separate fluid-like substance [25].

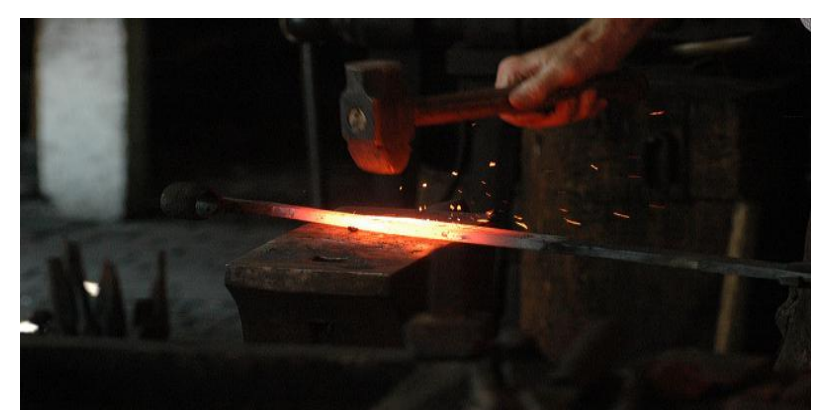

Fig. 2: Blacksmith working on hot iron [23].

We use the finite difference method (FDM) to find numerical solutions to heat transfer problems, which was introduced by Brook Taylor in 1715 and had been studied as abstract self-standing mathematical objects in works by George Boole (1860), C.M. Milne Thomson (1933) and Karaly Jordan (1939) [12, 19]. Makhtoumi [11] developed analytical and numerical solutions for studying heat diffusion investigating in a 1D thin rod. He used the rod PDE system to apply the homotopy perturbation method (HPM) and the finite difference method (FDM). Olaiju et al. [14, 15] investigated the explicit finite difference scheme and applied it to a simple 1D heat equation problem.

Finite difference methods are used to compute the numerical solutions of a one dimensional heat equation with initial and boundary conditions. To begin, the effect of heat supplied to the material at various positions will be investigated and compared. Finally, the effect of heat supplied in various positions will be investigated and compared.

\section{NUMERICAL SOLUTIONS FOR VARIATION OF INITIAL CONDITIONS}

\subsection{Heat Equation for 1D Iron Rod}

Let us consider an example of a one dimensional heat equation in the case of iron rod, whose thermal 
diffusivity is $0.23 \mathrm{~cm}^{2} / \mathrm{s}=0.000023 \mathrm{~m}^{2} / \mathrm{s}$ at $26.85^{\circ} \mathrm{C}$ temperature [21] as follows

$u_{t}=0.000023 u_{x x}, \quad 0 \leq x \leq 1, \quad t \geq 0 \ldots$ (2.1)

BCs: $u(0, t)=u(1, t)=0 ; \quad t>0$

And for the different experiments we apply three different initial conditions.

\subsubsection{Numerical Solution by Using FDM}

The FTCS scheme of the above heat equation is [6]

$$
v_{m}^{n+1}=v_{m}^{n}+c\left(v_{m+1}^{n}-2 v_{m}^{n}+v_{m-1}^{n}\right)
$$

where, $v_{0}^{n}=v_{m}^{n}=0$

This FTCSS is consistent with the order of accuracy $(1,2)$ and is stable iff $c \leq \frac{1}{2}[6,20]$. We can maintain the condition of stability by resizing the lengths of space and time intervals. To find the more accurate approximation we have to increase the number of space and time partitions $[6,20]$. Let the length of space and time intervals be $h=0.1$ and $k=0.2$ respectively. Then, $c=\frac{0.000023 \times k}{h^{2}}=\frac{0.000023 \times 0.2}{0.1^{2}}=0.00046$

We know that, the FTCS scheme is stable iff $c \leq 0.5$, so our FTCS is stable for above problem.

\section{A: Variation of position of initial condition:}

In Nepali culture, blacksmith supplied heat at different position on the iron to make it fixable. Firstly, we discuss the variation position of initial condition, i.e., left end, middle and right end of the rod with heat equation (2.1) as above. Consider, a blacksmith applies the $1500 C^{o}$ temperature near left end, at middle and near right end of the rod of $1 \mathrm{~m}$ length successively. Thus, the corresponding initial conditions are:

$\mathrm{IC} 1: u(0.1,0)=1500$

IC2: $u(0.5,0)=1500$

IC3: $u(0.9,0)=1500$

After then we study the nature of heat transfer in the rod. The temperature distribution corresponding to the above three cases are shown in Fig. 3.
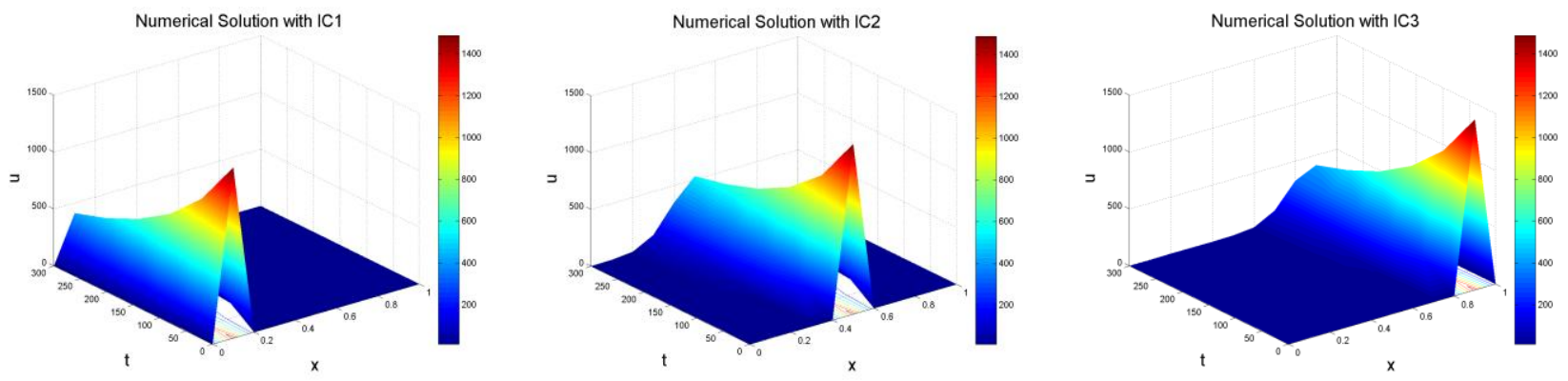

Fig. 3: Temperature distribution corresponding to Top: IC1, Middle: IC2 and Bottom: IC3.

From Fig. 3 we observe that, the figure with IC1 and IC3 allows heat to transfer in only one direction, to the right and left sides respectively. Heat, on the other hand, follows in either direction with IC2. A plot of the $\mathrm{x}$ versus temperature distribution is also shown below in Fig. 4.

This plot shows that after $250 \mathrm{sec}$. of heat, the iron rod is around 600 degrees Celsius, but in the first and third cases, the rod is only around 500 degrees Celsius. This variation is caused by the boundary conditions, which are set to $0^{\circ} \mathrm{C}$ at both ends. Heat applied to both the near left and near right produces the same result. However, the outcome would be more interesting if the material was nonhomogeneous or if the boundary conditions on the left and right ends differed.

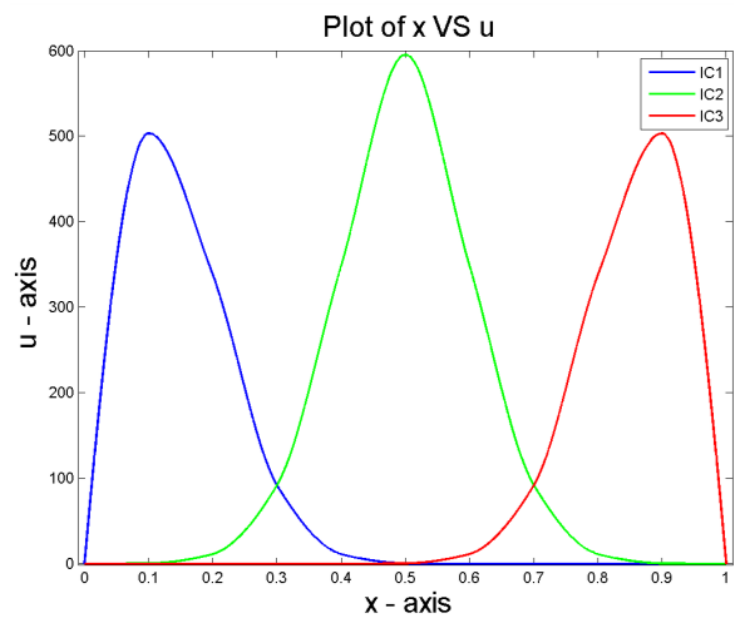

Fig. 4: Plot of $x$ versus u after $250 \mathrm{sec}$. of heat supplied 
B: Initial condition in Multiple Position: Here, the above concept has been generalized for the multiple initial conditions. Now for the first initial condition (IC1), we have

$$
v_{m}^{0}=\left\{\begin{array}{c}
1500 \text { for } m=5 \\
0 \text { otherwise }
\end{array}\right.
$$

Similarly, for the initial conditions IC2 and IC3 respectively, we have

$$
\begin{aligned}
& v_{m}^{0}=\left\{\begin{array}{cl}
1500 & \text { for } m=3,7 \\
0 & \text { otherwise }
\end{array}\right. \text { and } \\
& v_{m}^{0}=\left\{\begin{aligned}
1500 & \text { for } m=2,5,8 \\
0 & \text { otherwise }
\end{aligned}\right.
\end{aligned}
$$

Fig. 5 depicts the temperature distribution with different initial conditions, namely IC1, IC2 and IC3, up to $300 \mathrm{sec}$. from the instant of heating the material. According to Fig. 5, the temperature of the material, i.e., iron rod, gradually goes on decreasing from the time of heating. Over time, the rod cooled down to around $500^{\circ} \mathrm{C}$ from $1500^{\circ} \mathrm{C}$ in just $300 \mathrm{sec}$.

In IC1, we heated the road at one point, in IC2, we heated it at two points and in IC3, we heated at three different points, as stated above. The front edge of the numerical solution with IC3 in Fig. 5 is browner and darker red. This demonstrates that the rod has a more even temperature distribution than IC1 and IC2. This is because the number of heatsupplied locations has increased. Figure 2 depicts a real-world application of one of the blacksmiths' iron road heating techniques. This method of heating iron rods and other materials was also used by various metal industries, including blacksmiths and goldsmiths.
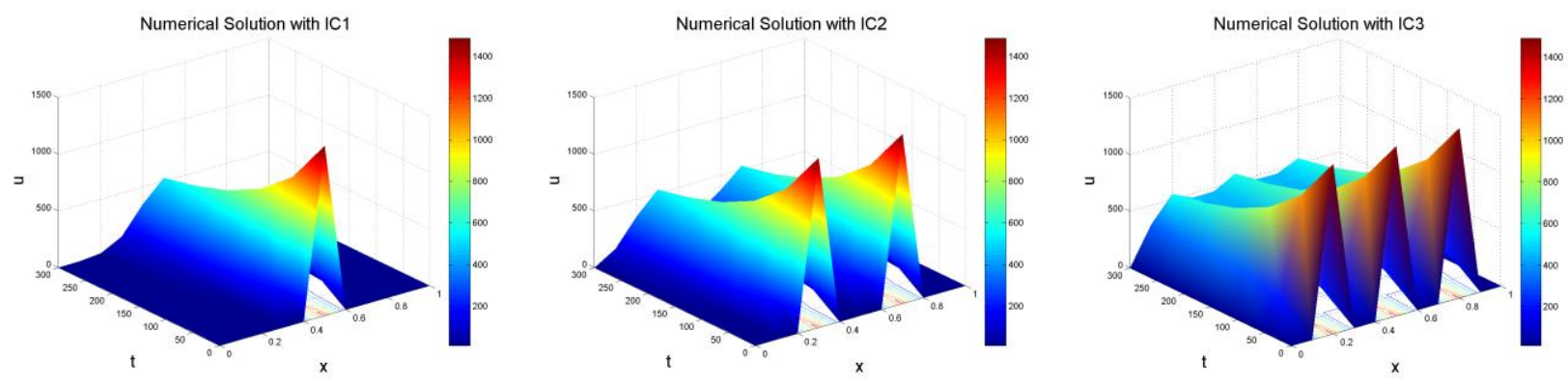

Fig. 5: Numerical solution of (2.1) with different initial conditions.

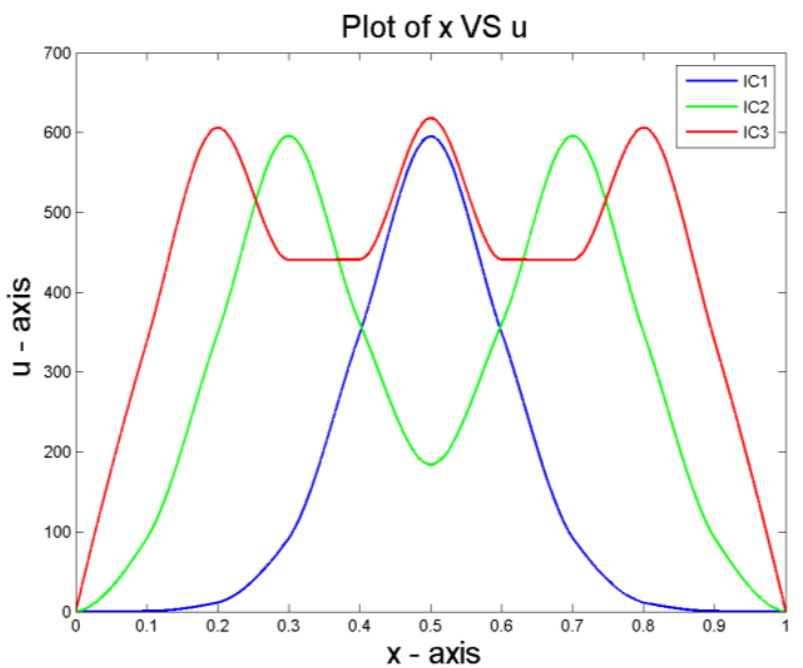

Fig. 6: Plot of $x$ versus u after $250 \mathrm{sec}$. of heat supplied.

According to Fig. 6, the average temperature after $250 \mathrm{sec}$. of heating the iron rod is around $590^{\circ} \mathrm{C}$. The figure shows that the amount of average temperature on the rod is greater with IC3 than that of IC2 and IC1 as a consequence of applying heat in more places than that of IC1 and IC2. As a result, we conclude that, the average temperature of the iron rod is proportional to the number of heat applied locations.

\section{CONCLUSION}

Using FDMs, we compute the numerical solution of one dimensional heat equation. To begin, we consider a one dimensional heat equation and consider the variety of positions of initial conditions, that is, heat is applied at the left, middle, and right ends of the rod. Initially, we applied the $1500^{\circ} \mathrm{C}$ temperature to the aforementioned positions of the $1 \mathrm{~m}$ long rod in succession. The nature of the heat transfer in the rod is then investigated. We discovered that when the temperature is applied to the center of the rod, it lasts longer. The outcome is due to the boundary conditions we used. Similarly, we applied $1500^{\circ} \mathrm{C}$ 
temperature to one position, then two positions, and finally three positions of the one dimensional iron rod. We conclude from this experiment that the average temperature of the iron rod is directly proportional to the number of heat applied locations. This method of heating iron rods and other materials was used by various metal industries, including blacksmiths and goldsmiths.

\section{REFERENCES}

[1] Azmi, A. Z. Numerical Solution for Heat Equation. Diss. Universiti Teknologi Malaysia, (2009).

[2] Boyce, W. E.; DiPrima, R. C. and Meade, D. B. Elementary Differential Equations and Boundary Value Problems, Loose-Leaf Print Companion. John Wiley \& Sons (2017).

[3] Chamkha, A. J. and Khaled, A. A. Hydromagnetic combined heat and mass transfer by natural convection from a permeable surface embedded in a fluid-saturated porous medium. Int. J Numer. Meth. H., 12(8): 234-250 (2000).

[4] Dabral, V.; Kapoor, S. and Dhawan, S. Numerical Simulation of one dimensional Heat Equation: BSpline Finite Element Method. Ind. J. Comp. Sci. Eng., 2(2): 222-235 (2011).

[5] Hayat, T.; Kara, A. H. and Momoniat, E. The unsteady flow of a fourth-grade fluid past a porous plate. J. App. Mat. Comp., 200: 65-76 (2005).

[6] Kafle, J.; Bagale, L. and K. C., D. Numerical Solution of Parabolic Partial Differential Equation by Using Finite Difference Method. $J$. Nep. Phys. Soc., 6(2): 57-65 (2020).

[7] Kafle, J.; Thakur, B. K. and Bhandary, I. Visualization, Formulation and Intuitive Explanation of Iterative Methods for Transient Analysis of Series RLC Circuit. BIBECHANA, 18(2): 2382-5340 (2021).

[8] Kattel, P. and Kafle, J. Differential Equation with Application (for BSc Second year), Sukunda Pustak Bhawan, Bhotahity, Kathmandu (2018).

[9] Kazem, S. and Dehghan, M. Application of finite difference method of lines on the heat equation. Wil. Per., (2017).

[10] KC, D. J. and Budhathoki, T. B. A Textbook of Differential Equation (for BSc third year), Heritage Publishers and Distributors Pvt. Ltd, Bhotahity, Kathmandu (2018).

[11] Makhtoumi, M. Numerical Solutions of Heat Diffusion Equation Over One Dimensional Rod Region. Int. J. Sci. Adv. Tec., 7(3): 10-13 (2017).

[12] Mebrate, B. Numerical Solution of a One Dimensional Heat Equation with Dirichlet
Boundary Conditions. Amer. J. Appl. M., 3(6): 305-311 (2015).

[13] Narasimhan, T. N. Fourier's Heat Conduction Equation: History, Influence, and Connections, Law. Nat. Lab. (1999).

[14] Olaiju, O. A.; Hoe, Y. S. and Ogunbode, E. B. Finite Difference Approximations to the Heat Equation via C. J. Appl. Sci. Env., 3(7): 188-200 (2017).

[15] Olaiju, O. A.; Hoe, Y. S. and Ogunbode, E. B. Finite Element and Finite Difference Numerical Simulation Comparison for Air Pollution Emission Control to Attain Cleaner Environment. Chem. Eng. Trans., 63: 679-684 (2018).

[16] Paudel, K.; Bhandari, P. and Kafle, J. Analytical Solution for Advection-Dispersion Equation of the Pollutant Concentration using Laplace Transformation. J. Nep. Math. Soc.., 4(1): 33-40 (2021).

[17] Pokhrel, P.; Lamsal, B.; Kafle, J. and Kattel, P. Analysis of displacement of vibrating of massspring due to opposition force. Tri. Univ. J., 35(1): 21-32 (2020).

[18] Pokhrel, P. R.; Kafle, J.; Kattel, P. and Gaire, H. Analysis of blood flow through artery with mild stenosis. J. Ins. Sci. Tech., 25(2): 33-38 (2020).

[19] Reddy, J. N. Solutions Manual for An Introduction to The Finite Element Method, Fourth Edition, McGraw-Hill, New York, 420 (2006).

[20] Strikwerda, J. C. Finite Difference Schemes and Partial Differential Equations, 2nd ed. (2004).

[21] Wilson, J. Materials Data, Electronics Cooling (2007).

[22] https://en.wikipedia.org/wiki/Blacksmith

[23] https://en.wikipedia.org/wiki/Blacksmith\#/media/ File:Blacksmith working.jpg

[24] www.academia.edu/35774098/Chapter-01History-of-Heat-Equation.

[25] www.wolframscience.com/reference/notes/1019b 